between Xpert SA and the culture results. Methicillin resistance was determined using conventional methods (susceptibility testing or detection of altered penicillin binding protein).

Results. When compared with culture for the identification of SA $(n=481)$, there was an agreement of $95.0 \%$ with sensitivity and specificity being $95.6 \%$ and $94.8 \%$, respectively. Among those culture-confirmed and Xpert SA positive samples $(n=131)$, concordance between Xpert SA and conventional methods for detection of methicillin resistance was $97.0 \%$ with sensitivity and specificity being $100 \%$ and $96.3 \%$, respectively. Four culture-confirmed methicillin-susceptible SA (MSSA) were identified as MRSA by Xpert SA. Among 504 nasal specimens, 23 (4.6\%) samples had invalid or instrument failure results. Nasal swabs collected from pediatric patients ( $\leq 21$-year-old) had a higher invalid/instrument failure rate $(5.0 \%)$ than those from adults $(0 \%)(P<$ 0.001 ).

Conclusion. Xpert SA Nasal Complete assay provides a rapid and sensitive method to detect and differentiate between MSSA and MRSA colonization. The higher invalid rate in pediatric patients and misidentification of MSSA as MRSA by Xpert SA warrant the confirmation by bacterial culture and conventional susceptibility test.

Disclosures. A. Leber, Nationwide Children's Hospital: Research Contractor, Research support.

2005. T-SPOT .TB Test for Latent Tuberculosis Infection Diagnosis and Treatment Guidance in Thai Healthcare Professionals

Thana Khawcharoenporn, MD, MSc ${ }^{1}$; Waralee Aksornchindarat, $\mathrm{BSc}^{2}$; Napat Yodpinij, $\mathrm{BSc}^{2}$; Sopa Srisungngam, $\mathrm{BSc}^{3}$; Janisara Rudeeaneksin, $\mathrm{BSc}^{3}$; Supranee Bunchoo, BSc ${ }^{3}$; Wiphat Klayut, $\mathrm{BSc}^{3}$; Somchai Sangkitporn, $\mathrm{MD}^{3}$ and Benjawan Phetsuksiri, $\mathrm{PhD}^{3}$; ${ }^{1}$ Department of Medicine, Faculty of Medicine, Thammasat University, Pathumthani, Thailand, ${ }^{2}$ Thammasat University, Pathumthani, Thailand, ${ }^{3}$ National Institute of Health, Ministry of Public Health, Nonthaburi, Thailand

Session: 228. Diagnostics: Bacteria and Mycobacteria Saturday, October 6, 2018: 12:30 PM

Background. Data on efficacy of T-SPOT ${ }^{\circ}$.TB Test (T-SPOT) in diagnosing latent tuberculosis infection (LTBI) and guiding isoniazid preventive therapy (IPT) among healthcare professionals (HCP) in tuberculosis (TB)-endemic settings are limited.

Methods. A prospective study was conducted among Thai HCP undergoing T-SPOT in June 2016 (initial screening) and June 2017 (follow-up). Ninemonth isoniazid preventive therapy (IPT) was offered among the HCP with positive T-SPOT. The incidence of TB and the rates of conversion and reversion of T-SPOT were evaluated during the 1-year follow-up period (June 2016 to June 2017).

Results. A total of $140 \mathrm{HCP}$ underwent initial T-SPOT; the median age was 27 years (IQR 25-31 years), 89\% were female and 23 (16\%) were T-SPOT-positive. Eighty-nine HCP (64\%) had both initial and follow-up T-SPOTs. Among the 89 HCP, the initial and follow-up rates of T-SPOT positivity were $19 \%(N=17)$ and $24 \%(N=21)$, respectively. The conversion and reversion rates were $10 \%(N=9)$ and $6 \%(N=5)$, respectively. All of the nine HCP $(100 \%)$ with T-SPOT conversion reported significant contacts with patients who had active pulmonary TB without using appropriate personal protection equipment. During the 1-year follow-up period, incidence of TB were significantly higher among HCP with T-SPOT conversion compared with HCP with persistent positive T-SPOT, HCP with T-SPOT reversion and HCP with persistent negative T-SPOT [ 22 vs. 8 vs. 0 vs. 0 cases/100 person-years; $P<0.001]$. Of the $17 \mathrm{HCP}$ with positive initial T-SPOT, $8(47 \%) \mathrm{com}$ pleted IPT. The incidence of TB was significantly lower and the T-SPOT reversion rate was significantly higher among HCP completing IPT compared with HCP declining or not completing IPT ( 0 vs. 11 cases/100 person-years; $P<0.001$ and $63 \%$ vs. $0 \% ; P=0.009$, respectively).

Conclusion. T-SPOT could be used for diagnosing LTBI, guiding IPT and identifying HCP with subsequent risk for TB. The serial T-SPOT may be used for evaluating IPT efficacy.

Disclosures. All authors: No reported disclosures.

\section{Implementation of the T2 Biosystems T2Bacteria Panel in a Level-One Trauma Center, Safety Net Hospital \\ Caitlin Robinson, $\mathrm{BS}^{1}$; Rebecca Jackson, $\mathrm{MS}^{1}$; Angela Sauaia, $\mathrm{MD}, \mathrm{PhD}^{1,2}$ and Mitchell Cohen, $\mathrm{MD}^{1,3}$; ${ }^{1}$ Surgery, Denver Health and Hospital Authority, Denver, Colorado, ${ }^{2}$ Public Health and Surgery, University of Colorado Anschutz Medical Campus, Aurora, Colorado, ${ }^{3}$ Biomedical Informatics and Personalized Medicine, University of Colorado Anschutz Medical Campus, Aurora, Colorado \\ Session: 228. Diagnostics: Bacteria and Mycobacteria \\ Saturday, October 6, 2018: 12:30 PM}

Background. Rapid detection and identification of sepsis causing pathogens are critical for optimizing antimicrobial therapy to improve patient survival and reduce healthcare costs. The T2Bacteria Panel RUO is a molecular diagnostic allowing detection of Gram-negative Escherichia coli, Klebsiella pneumoniae, Pseudomonas aeruginosa, Acinetobacter baumannii and Gram-positive Staphylococcus aureus and Enterococcus faecium within a few hours. The purpose of our study was to determine the feasibility and efficacy of the T2Bacterial Pane RUO in an Emergency Medicine (ED) and Surgical Intensive Care Unit (SICU) setting.

Methods. An IRB-approved, prospective, observational study was implemented at a Safety-Net, Level One Trauma Center in Denver, Colorado. Patients were enrolled who received an order for a blood culture from the ED or SICU. Patients who had blood drawn for cultures had a concurrent draw for testing with a T2Bacteria Panel RUO.

Results. Sixty-six patients are included in the present interim analysis. Mean patient age was 51 years old (19-84), 36\% were female, 86\% Caucasian (34\% Hispanic/ Latino), and $74 \%$ of patients were enrolled upon presentation to the ED, $13 \%$ from the SICU, and $15 \%$ from the wards. $90 \%$ of blood sampling (culture and T2Bacteria) was done from peripheral stick while $7 \%$ were from the initial stick of a peripheral IV and $2 \%$ obtained from an indwelling catheter. $85 \%$ of blood cultures were negative. Of the 56 patients with negative blood culture, 53 had concordant negative T2Bacteria results, providing a specificity of $94.6 \% .10$ patients had positive blood cultures $(15 \%)$ for T2Bacteria Panel RUO targets. Interestingly, only five of these (50\%) had concordant positive T2Bacteria testing. Examining the discordant samples, all (5) blood culture positive, T2Bacteria negative were found to have clinically false-positive blood cultures. T2Bacteria positive samples were distributed as follows: two E.coli, one S. aureus, one K. pneumoniae, and one P. aeruginosa. No detections were made for E. faecium or A. baumannii.

Conclusion. In this interim analysis, T2Bacteria Panel RUO provides feasible rapid diagnostics for ED and surgical ICU settings with a high specificity and much shorter time to result when compared with gold standard blood cultures.

Disclosures. C. Robinson, T2 Biosystems: Research Coordinator for funded study from T2 Biosystems, Research support. R. Jackson, T2 Biosystems: Research Assistant for funded study from T2 Biosystems, Research support. M. Cohen, T2 Biosystems: PI on study being funded by T2 Biosystems, not paying for any part of salary, Research support.

2007. To Treat or Not to Treat: Does a More Sensitive and Specific Testing Methodology Make the Treatment Decision More Clear?

Bakri Kulla, MD, ID ${ }^{1}$; Patrick Haggerty, MD, ID ${ }^{2}$; Shivanjali Shankaran, MD $^{3}$; Hadiza Galadima, $\mathrm{PhD}^{4}$ and Matthew Peworchik, $\mathrm{NA}^{5} ;{ }^{1}$ Eastern Virginia Medical, Norfolk, Virginia, ${ }^{2}$ Eastern Virginia Medical School, Norfolk, Virginia, ${ }^{3}$ Infectious Diseases, Eastern Virginia Medical School, Norfolk, Virginia, ${ }^{4}$ Sentara Healthcare Analytics and Delivery Science Institute (HADSI), Eastern Virginia Medical School, Norfolk, Virginia, ${ }^{5}$ Eastern Virginia Medical School, Norfolk, Virginia

Session: 228. Diagnostics: Bacteria and Mycobacteria

Saturday, October 6, 2018: 12:30 PM

Background. Clostridium difficile infection (CDI) is a leading cause of infectious diarrhea in healthcare settings in the United States. Accurate testing methodology provides guidance to clinicians as to when to treat. Our study was designed to determine whether more sensitive testing methodology implemented in 2013 reduced unnecessary treatment of hospital associated diarrhea (HAD).

Methods. In 2012, patients with HAD were tested with the less sensitive testing method of C. difficile Toxin Assay by EIA. In 2013, a three-step algorithm incorporated $\mathrm{CDI}$ glutamate dehydrogenase antigen (GDH) in combination with an enzyme-linked immunoassay for Toxin A and B was introduced. Those samples with discrepant results (positive on only one of the two) were considered indeterminate and subjected to the nucleic acid amplification test (NAAT) for CDI genes. In a retrospective chart review of HAD, we assessed the decision to treat based on the laboratory results available at the time in the pre-algorithmic and post-algorithmic periods. Multiple demographic factors and comorbid conditions were analyzed to provide clues to why the patient may have had continued treatment despite negative assays.

Results. The rate of treated patients despite negative CDI testing in the pre-algorithm period was $59 \%(118 / 444)$ and $41 \%(82 / 249)$ in the post-algorithm period $(P$ $=0.0765)$. A multiple logistic regression analysis was done for all tested factors. The factors that led to treatment despite negative testing in both time periods included: organ transplantation $(P=0.0003)$, other immunosuppressive conditions $(P=0.0447)$, prior hx of CDI $(P=0.0021)$, longer length of stay $(P=0.0105)$, and hx of hypertension $(P=0.0173)$.

Conclusion. While there was a downward trend toward holding CDI treatment in those with negative CDI testing as the more sensitive and specific algorithm was introduced it did not reach statistical significance. The higher risk patients were statistically more likely to be treated even if the testing was negative. Further efforts should be made to educate clinicians as to the accuracy of the testing methods so that appropriate antibiotic de-escalation can be achieved even in high-risk patients with diarrhea.

Disclosures. All authors: No reported disclosures.

2008. Effective and Early Diagnosis of Pneumonia in Patients With Acute Leukemia in a Comprehensive Cancer Center: How Can We Improve the Microbiological Diagnosis?

Aki Sakurai, MD ${ }^{1}$; Justin Bala-Hampton, DNP, MPH, APRN ${ }^{1}$; Victor E. Mulanovich, $\mathrm{MD}^{2}$; Jorge E. Cortes, $\mathrm{MD}^{3}$ and Javier Adachi, $\mathrm{MD}^{4}$; ${ }^{1}$ Infectious Diseases, The 